\title{
Long range, high resolution laser radar
}

\author{
A. Braun, C.Y. Chien, S. Coe and G. Mourou \\ Center for Ultrafast Optical Science, University of Michigan, 2200 Bonisteel, Ann Arbor, MI 48108-2099, USA
}

Received 2 June 1993; revised manuscript received 21 September 1993

\begin{abstract}
A high energy, chirped pulse laser was used to demonstrate long range, high resolution laser radar. A broadband nanosecond chirped pulse propagated to a target, and upon compression sub-millimeter surface resolution was obtained. This technique avoided the nonlinear effects in air that were seen when a picosecond pulse propagated to the target.
\end{abstract}

Initial laser radar experiments used $\mathrm{CO}_{2}$ gas lasers at $10 \mu \mathrm{m}[1]$. These lasers enabled either stable cw operation or high-gain pulsed output and have been used for wind measurements in the atmosphere. Despite the success of $\mathrm{CO}_{2}$ lasers, there has recently been an increased interest in the use of solid state, $1 \mu \mathrm{m}$ laser technologies to be used for laser radar studies. The advantages of such a system include high pulseto-pulse reliability, the availability of room temperature detectors, and the possibility of a compact diode pumped solid state system in the future. Several groups developing lasers radar used pulses from $\mathrm{Nd}$ :YAG systems with pulsewidths ranging from 1 $\mu$ s to $8 \mathrm{~ns}$ with pulse energies from $12 \mathrm{~mJ}$ to $1 \mathrm{~mJ}$, respectively [2-5]. These transform limited pulses were used to measure velocities from the Doppler shift or to perform range-resolved experiments. The spatial resolution achieved in these experiments was directly related to the rise time of the pulses generated from the laser. The minimum rise times used were a few nanoseconds, which corresponded to resolvable distanees within one meter [4]. To obtain range resolved measurements to within one millimeter, a modelocked laser producing pulses with a picosecond rise time would be needed. Picosecond pulses could also be used to measure the surface profile of the target with millimeter accuracy. Using the technique of chirped pulse amplification (CPA), picosecond pulses can be temporally stretched (chirped), safely amplified to the joule level, and compressed to their Fourier transform limit [6]. We have demonstrated that high energy broad band- width nanosecond chirped laser pulses can propagate to a target, and the return echo pulses can be compressed to their Fourier transform limit to obtain sub-millimeter surface resolution of the target. Propagation of the long chirped pulse instead of the compressed picosecond pulse avoided the small scale self-focusing and self-phase modulation effects in air and offers the possibility of a long range, high resolution laser radar system.

For this experiment, pulses were obtained from a CPA system [7] which had an uncompressed chirped time duration of $1.2 \mathrm{~ns}$ with $400 \mathrm{~mJ}$ of energy and a rise time of one nanosecond, and a compressed pulsewidth of $400 \mathrm{fs}$ with $250 \mathrm{~mJ}$ of energy and a rise time of $300 \mathrm{fs}$. The pulse's spectral width was $4.6 \mathrm{~nm}$ and was centered at $1.053 \mu \mathrm{m}$. To obtain the highest spatial resolution possible, it was desired to propagate the $400 \mathrm{fs}$ pulse to the target. However, the power of this pulse was large enough to cause beam filamentation. The critical power for self-focusing can be calculated from the equation [8]:

$P_{\text {cr }}=\pi(0.61)^{2} \lambda^{2} / 8 n_{0} n_{2}$,

where $n_{2}$ is the nonlinear index of refraction. Using $n_{2}=0.56 \times 10^{-18} \mathrm{~cm}^{2} / \mathrm{W}$, the nonlinear index of air, $P_{\mathrm{cr}}$ is $3 \mathrm{GW}$. Since the peak power of the compressed pulse was $0.6 \mathrm{TW}$, beam filamentation would occur as the pulse propagated in air. These filaments would undergo an exponential growth with a gain coefficient given by [9]

$g=K\left(\frac{n_{2} I}{n_{0}}-\frac{K^{2} \lambda^{2}}{16 \pi^{2}}\right)^{1 / 2}$ 
where $K$ is the spatial wave number of the modulation on the beam and $I=120 \mathrm{GW} / \mathrm{cm}^{2}$. For the case of propagation in air, with the measured $K$ of $1 / \mathrm{mm}$, the filaments would grow with a gain coefficient of $g=0.245 / \mathrm{m}$. It can be shown that these filaments would reach a focus after a propagation length given by [9]

$Z_{\mathrm{f}}=(g)^{-1} \ln (3 / \delta)$,

where $\delta$ is the modulation depth on the beam. For our case $\delta$ was measured to be $30 \%$, and $Z_{\mathrm{f}}$ was calculated to be $9.4 \mathrm{~m}$. It was also expected that selfphase modulation would become important if the spectrum broadened by an amount equal the spectral width of the pulse. This would occur after a propagation length of [10]

$L_{\text {spm }}=(2 \Delta \lambda / \lambda)\left(c T_{0} / n_{2} I\right)$,

where $T_{0}$ is the pulsewidth and $\Delta \lambda$ is $4.6 \mathrm{~nm}$. From this equation, a propagation distance of $15.6 \mathrm{~m}$ was calculated.

Self-focusing would not develop if the uncompressed pulse propagated in air because its power was 10 times below the critical power $(0.33 \mathrm{GW})$. Furthermore, as $\mathrm{d} I / \mathrm{d} t$ was $6 \times 10^{6}$ times less for the uncompressed pulse as compared to the compressed pulse, the uncompressed pulse could propagate $94 \times 10^{6} \mathrm{~m}$ in air before self-phase modulation would become important. Propagating the nanosecond pulse to the target would avoid the nonlinear effects in air, and compressing the return pulses would ideally give $120 \mu \mathrm{m}$ spatial resolution of the target.
A schematic of the experimental setup to propagate the compressed pulse is shown in fig. 1. The target was placed $33 \mathrm{~m}$ from the laser. The target consisted of a $50 \%$ beam splitter slightly off normal to the beam (coated side away from the beam) and a high reflector behind and parallel to the beam splitter. The high reflector was placed on a linear translation stage to allow a variable time delay between the second return pulse with respect to the first reflected pulse. In addition to a small delay between the two return pulses, this arrangement enabled both pulses to traverse the same amount of glass. The two return pulses were aligned into a single-shot autocorrelator where their measured temporal separation was directly related to the spatial separation of the reflectors. This optical detection technique was used because electronic detectors cannot respond fast enough to accurately resolve the picosecond time delay between the return pulses. To obtain a reference autocorrelation trace for the case of propagating a compressed pulse, the pulse energy was reduced to $4 \mathrm{~mJ}$. As is seen in fig. $2 \mathrm{a}$, a clearly resolvable autocorrelation trace was measured which corresponded to a reflector separation of $400 \mu \mathrm{m}$. This distinct autocorrelation trace became washed out when the pulse energy was increased to $250 \mathrm{~mJ}$. Figure $2 \mathrm{~b}$ shows an autocorrelation trace corresponding to the same reflector separation with a peak power of $0.6 \mathrm{TW}$. As can be seen, the high resolution has been lost due to nonlinear effects experienced in the air and glass. Filamentation of the beam was observed after about ten meters of propagation in air. Also, the spectrum broadened by a factor of 3.5 due

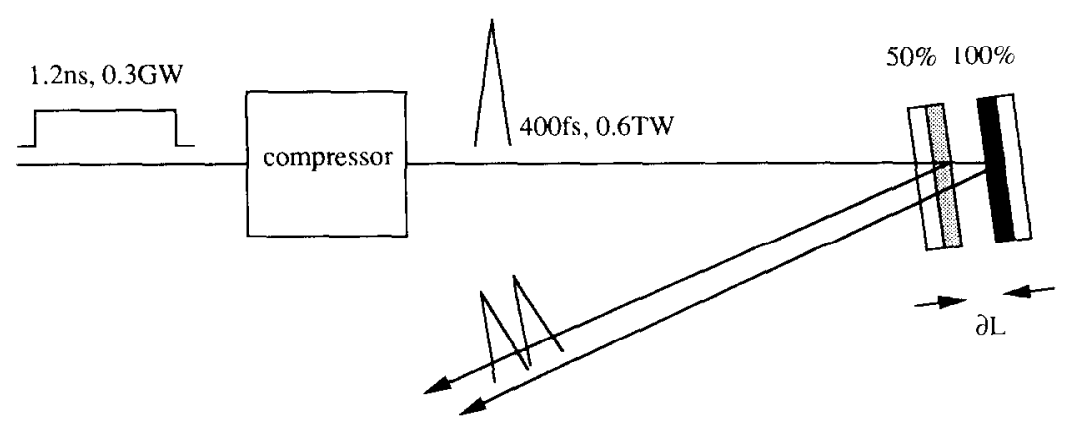

To Autocorrelator

Fig. 1. Schematic of experimental setup to propagate the compressed pulse to the target. The two return pulses were aligned through a single shot autocorrelator to determine the separation between the two reflectors. 

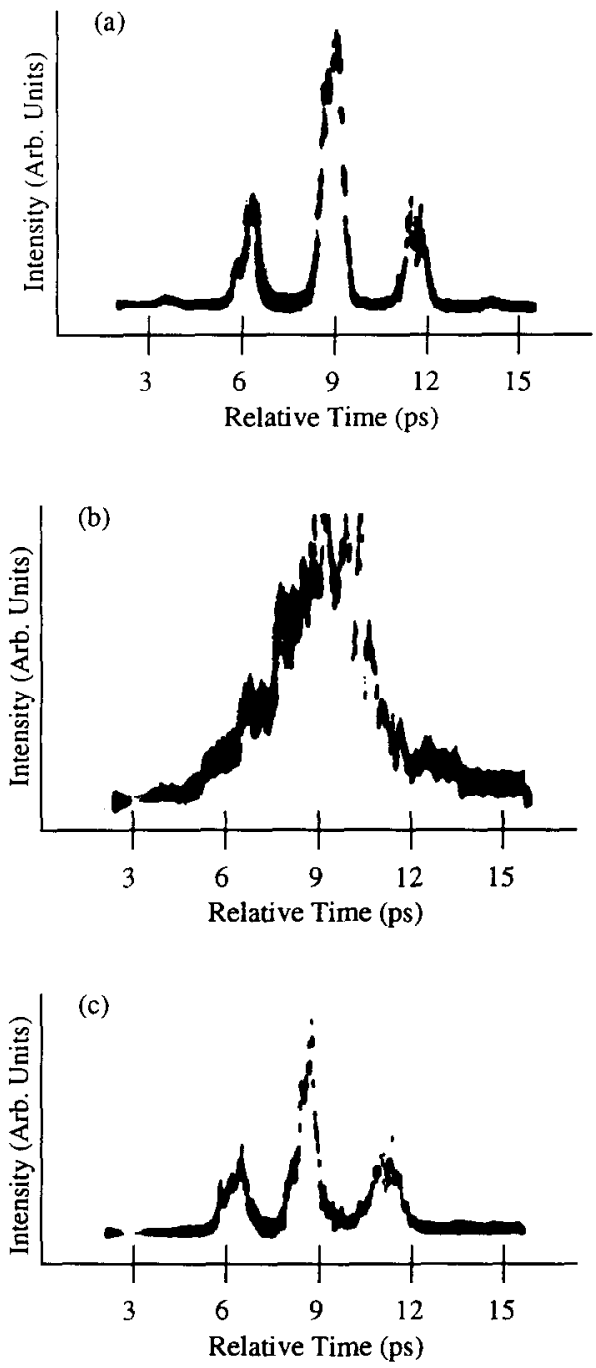

Fig. 2. (a) After propagating a compressed pulse with $4 \mathrm{~mJ}$ of energy to the target, a reflector separation of $400 \mu \mathrm{m}$ was obtaincd. (b) Autocorrclation trace of two return pulses after propagating a compressed pulse with $250 \mathrm{~mJ}$ of energy. The reflector separation was still $400 \mu \mathrm{m}$, but the resolution was lost due to nonlinear effects experienced in air. (c) After propagating a nanosecond chirped pulse with $400 \mathrm{~mJ}$ of energy to the target, the return pulses were compressed and sent into an autocorrelator to show a reflector separation of $360 \mu \mathrm{m}$.

to self-phase modulation after about $40 \mathrm{~m}$ of propagation. These experimental observations correspond fairly well to the theoretical values stated above.

The same experiment was then performed by by- passing the compressor and propagating the nanosecond, chirped pulse to the target with the two chirped return pulses directed into the compressor. To measure the reflector's separation, the two compressed pulses were aligned into the single-shot autocorrelator as before. A schematic of the experimental setup is shown in fig. 3. The return pulses were compressed to their Fourier transform limit in order to be able to clearly resolve the peaks in the autocorrelation trace. If the uncompressed return pulses were sent into the single shot autocorrelator, the picosecond delay between the pulses would be indistinguishable in the nanosecond background. The propagation distance from the laser to the target was extended from $33 \mathrm{~m}$ to $68 \mathrm{~m}$. The pulse traveling to the target contained $400 \mathrm{~mJ}$ of energy and had a peak power of $0.3 \mathrm{GW}$. Figure $2 \mathrm{c}$ shows the autocorrelation trace of the two return pulses out of the compressor. This trace was clearly resolvable and corresponded to a reflector separation of $360 \mu \mathrm{m}$. By propagating a lower peak power pulse, high resolution was achieved even with the maximum energy per pulse. This demonstrated that the nanosecond, chirped pulse experienced negligible distortion when propagating through air and glass, and that upon compression excellent resolution could be obtained.

Through the technique of chirped pulse amplification, pulses can be generated which contain the necessary energy to propagate long distances used for laser radar studies. Furthermore, which the broad bandwidth chirped pulse comes the ability to compress the return echoes to obtain extremely high spatial resolution of the target. For less reflective targets, a more sensitive picosecond streak camera could be used instead of the single-shot autocorrelator to measure the delay between the return pulses. This process could also be used to obtain millimeter range resolution by comparing the time delay between a local reference pulse and a pulse that has been reflected back from the target. This technique could be used for a variety of applications including atmospheric boundary layer measurements, high accuracy moon or satellite ranging measurements, or ocean layer and oil slick thickness measurements. Even though the filamentation of the compressed pulse was not useful for this experiment, the intense filaments could be used to cause breakdown in air to produce laser guided lightning [11]. 


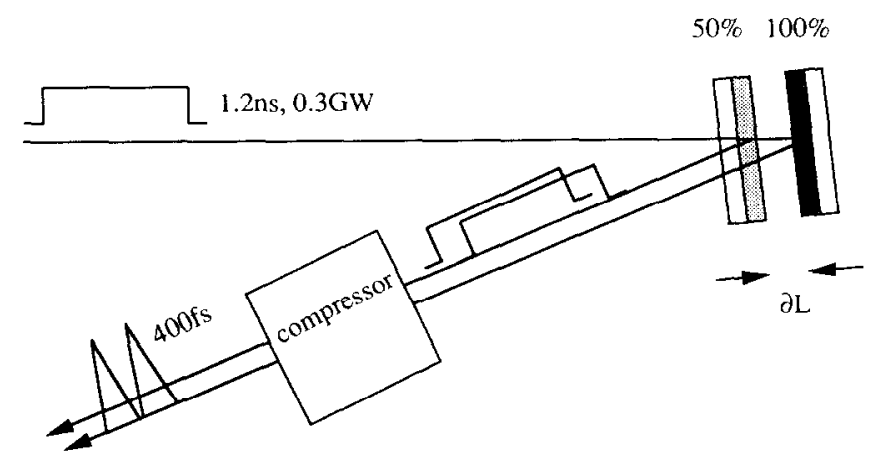

To Autocorrelator

Fig. 3. Schematic of experimental setup to propagate a nanosecond chirped pulse to the target. The two chirped return pulses were directed through a compressor before being aligned into an autocorrelator.

This work was supported by the National Science Foundation through the Center for Ultrafast Optical Science under STC PHY 8920108.

\section{References}

[1] R.T. Menzies and R.M. Hardesty, Proc. IEEE 77 (1989) 449.

[2] T.J. Kane, W.J. Kozlovky, R.L. Byer and C. Byvik, Optics Lett. 12 (1987) 239.

[3] M.J. Kavaya, S.M. Henderson, J.R. Magee, C.P. Hale and R.M. Huffaker, Optics Lett. 14 (1989) 776.

[4] K.P. Chan and D.K. Killinger, Opt. Eng. 30 (1991) 49.
[5] J.L. Bufton, J.B. Garvion, J.F. Cavanaugh, L. RamosIzquirdo, T.D. Clem and W.B. Krabill, Opt. Eng. 30 (1991) 72.

[6] D. Strickland and G. Mourou, Optics Comm. 56 (1985) 219.

[7] Y. Beaudoin, C.Y. Chien, J.S. Coe, J.L. Tapie and G. Mourou, Optics Lett. 17 (1992) 865.

[8] R.W. Boyd, Nonlinear optics (Academic, Boston, 1992) p. 260.

[9] W. Koechner, Solid-state laser engineering (Springer, Berlin, $1988)$ p. 547.

[10] R.W. Boyd, Nonlinear optics (Academic, Boston, 1992) p. 277.

[11] X.M. Zhao, C.Y. Yeh, J.-C. Diels and C.Y. Wang, in: Ultrafast phenomena VIII, eds. J.-L. Martin, A. Migus, G.A. Mourou and A.H. Zewail (Springer, Berlin, 1993) p. 264. 